\title{
Mesenchymal stem cell response to growth factor treatment and low oxygen tension in 3-dimensional construct environment
}

\author{
Thomas John Sakrison Durant \\ Nathaniel Dyment \\ Mary Beth R. McCarthy \\ Mark P. Cote \\ Robert A. Arciero \\ Augustus D. Mazzocca \\ David Rowe
}

Department of Orthopaedic Surgery, UCONN Health Center, Farmington, USA

Corresponding author:

Thomas John Sakrison Durant

Department of Orthopaedic Surgery

UCONN Health Center

Farmington, CT 06034, USA

E-mail: sakrisondurant@student.uchc.edu

\section{Summary}

Introduction: cell-based tissue engineering strategies using human mesenchymal stem cells (hMSCs) may help to augment tendon healing. To further investigate the in-vitro behavior of this cell population, we investigated low oxygen culture levels, and growth factor supplementation and their effect on expression of tendon extracellular proteins and cell proliferation.

Materials and methods: bone marrow aspirate (BMA) was harvested during arthroscopic rotator cuff repair. Characterized hMSCs derived from BMA were incorporated into 3-dimensional tissue engineered constructs (TECs). TECs were analyzed by frozen sections with immunohistochemistry for cell density, collagen I and collagen III expression.

Results: growth factor stimulation and low oxygen increased cell density within TECs. Low oxygen and addition of growth factors to culture media demonstrated an increase in collagen I and III expression, both in ambient oxygen conditions and low oxygen conditions.

Conclusion: low oxygen and TGF $\beta 3$ demonstrated a positive effect on cell number, and type I and III collagen expression in 3D culture environments.

KEY WORDS: construct, low oxygen, mesenchymal stem cells, tendon, transforming growth factor beta.

\section{Introduction}

Tendon injury is commonly encountered in the orthopaedic clinic, and is estimated to cost the United States healthcare system approximately 30 billion dollars per year ${ }^{1}$. Due to an aging population, the prevalence of such disease is expected to increase, along with the number of clinical encounters seen in orthopaedic practice ${ }^{2}$. While surgical management is still considered gold standard treatment, it consistently yields variable results, with failure rates ranging from $38-94 \%{ }^{3}$. Failures in tendon repair lead to revision surgeries and prolonged courses of physical therapy, leading to financial strain on the healthcare system, and decreased quality of life for the patient ${ }^{4}$. As a result of these repeated findings, recent investigation has sought to develop novel therapies which may involve cell-based augmentation strategies to improve tendon healing ${ }^{1,5}$.

While the literature involving tendon physiology has seen many advancements in recent years, tendon injury and subsequent healing is an issue which remains poorly understood ${ }^{5}$. Literature investigating the healing process of tendon has demonstrated that native cellular and extracellular matrix organization is lacking after an injury, and thus, the ability to recreate normal strength and function following surgical repair is limited ${ }^{6,7}$. In addition, it is noted in histologic studies of injured tendon, that misaligned type III collagen predominates the majority of tendon repair tissue, rather than organized type I collagen found in healthy embryologically derived tendon ${ }^{8}$.

Following the observation of varied surgical outcomes, combined with the understanding that tendon biology is significantly altered following injury, a body of literature has emerged, focusing on methods in which the healing process of tendon may be augmented ${ }^{1,5}$. Of the proposed methods, cell based-therapies have remained on the forefront, with human mesenchymal stem cells (hMSCs) becoming central to this research having already shown promising results ${ }^{3}$. Human MSCs are multipotent cells, which have the ability to self-renew and differentiate into cell types which include osteoblasts, tenocytes, chondrocytes and adipocytes, and with current technology, can be harvested from nearly any tissue in the body ${ }^{9-11}$.

The potential benefit of hMSCs may arise with their direct application to a surgical site, which may serve to augment the normal physiologic healing response, and improve surgical outcomes 4,12 . Such applications have been investigated in animal models and have shown improved healing rates in anterior cruciate ligament (ACL) repair, rotator cuff repair (RCR), patellar tendon defects and achilles tendon repairs ${ }^{5,13}$. Biologic augmentation 
Mesenchymal stem cell response to growth factor treatment and low oxygen tension in 3-dimensional construct environment

with cell-based regenerative therapy (CBRT) strategies using hMSCs may prove useful for treating these complex injuries ${ }^{14}$. However, further understanding of how these cells behave in vitro is needed prior to clinical application, with emphasis on how to ensure tenogenic differentiation of this multipotent progenitor cell type.

Currently there is paucity in the literature regarding the response of hMSCs to variables that may be encountered when applied in vivo. A growth factor shown to influence tendon development and healing is transforming growth factor beta-3 (TGFß3), which has demonstrated the ability to increase expression of tenogenic markers in mesenchymal progenitors in 2dimensional cell culture models ${ }^{12,14}$. Numerous in vitro studies have shown TGF $\beta$ signaling to increase the expression of tendon collagens, including type I and III collagen ${ }^{9}$. In addition, Pryce et al. ${ }^{15}$ demonstrated a disruption in TGF $\beta$ signaling (TGF $\beta 2$-/-;TGF $\beta 3$-/-) in double mutant mouse embryos, results in agenesis of most tendons and ligaments throughout the axial and appendicular skeleton, indicating that TGF $\beta$ signaling plays a major role in embryonic tenogenesis.

Oxygen levels of in vitro culture conditions have also been shown to be a prominent regulator of hMSC lineage tendencies, and thought to be an important in vivo variable $^{16,17}$. Partial pressures of oxygen in most in vitro studies is approximately $140 \mathrm{mmHg}$, and is thought to be considerably higher than what is found in most all mammalian tissues ${ }^{18-20}$. The partial pressure of oxygen in arterial blood has been measured from 60-110 $\mathrm{mmHg}$, while that of bone marrow has been measured in animals and mathematically calculated between 27$49 \mathrm{mmHg}$, corresponding with an approximate oxygen tension of $4-7 \%^{19-22}$. Therefore, it has been conceived that many cells, including those derived from bone marrow aspirate, may behave differently in vitro at oxygen concentrations lower than the ambient $21 \% 21$. A number of in vitro studies have supported this theory, demonstrating that low oxygen culture conditions for hMSCs allows maintenance of their progenitor state, and appears to regulate their proliferation, viability, and their commitment to differentiation $17,23,24$.

The objective of this study was to compare the effect of growth factor supplementation and cell culture oxygen levels, on expression of hMSC tendon extracellular matrix proteins, proliferation, and rate of gel construct compaction in a 3-Dimensional fibrin-based model. The hypotheses are: (1) TGFß3 will have a positive net effect on collagen production, cell proliferation, and rate of gel compaction and (2) low oxygen culture environment will have a positive net effect on collagen production, cell proliferation, and rate of gel compaction.

\section{Methods}

The method described in this paper were considered best practice in regards to commonly considered standards for research protocols. Best efforts were made to make the following justifiable and appropriate for the procurement of the conclusions stated, and the results from which they were drawn ${ }^{25}$.

\section{Experimental design}

HMSC-incorporated fibrin gels $\left(1 \times 10^{6}\right.$ cells $\left./ \mathrm{ml}\right)$ were cultured in ambient $(21 \%)$ or low $(5 \%)$ oxygen conditions in cell culture media supplemented with either $10 \%$ FBS or $10 \%$ FBS +TGF $\beta 3$. Tissue engineered constructs (TECs) were divided into 4 treatment groups. Normal oxygen with and without TGF $\beta 3$ addition, and low oxygen with and without TGF $\beta 3$ addition ( $n=3$ per group). TGF $\beta 3$ was added at a concentration $10 \mathrm{ng} / \mathrm{ml}$ to the $10 \%$ FBS with each cell media change, which was done every 3 days.

\section{Isolation of adult hMSCs and expansion in culture}

Briefly, we describe the previously published technique of MSC harvest during arthroscopic rotator cuff repair (RCR) by Mazzocca et al. ${ }^{3}$. A 14-gauge aspiration needle was inserted in the medullary cortex at the bone cartilage junction of the proximal head of the humerus where the first suture anchor for the RCR would be placed, to collect a bone marrow aspirate (BMA). BMA was centrifuged to isolate the pink middle layer, which was then aspirated and plated in cell culture dishes in equal volume distributions. Cells were expanded in either ambient $\left(21 \% \mathrm{O}_{2}\right)$ or low oxygen $\left(5 \% \mathrm{O}_{2}\right)$ conditions, and passaged once prior to creation of TECs.

\section{D Tissue Engineered Construct (TEC) Creation}

HMSCs were incorporated into a fibrin gel at a concentration of $1 \times 10^{6}$ cells per ml. $400 \mu \mathrm{L}$ of cells, 90 $\mu \mathrm{L}$ of fibrinogen and $10 \mu \mathrm{L}$ of thrombin were mixed to create a fibrin gel. MSC gels in all groups formed a uniaxial construct by contracting around two segments of 0 gauge nylon suture within 6 -well plates (Figs. 1, 2). TECs were grown for a period of 14 days

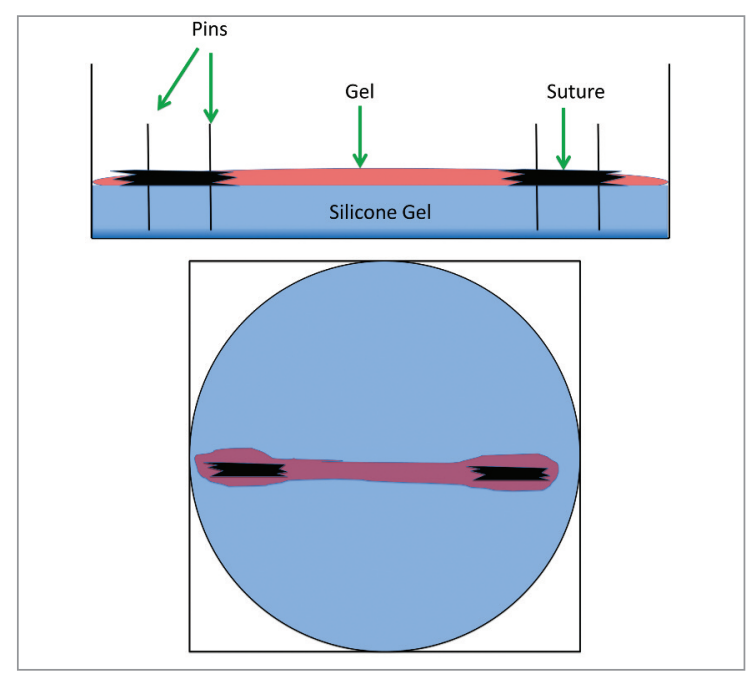

Figure 1. Drawings depicting lateral and top views (left to right) of tissue engineered constructs in silicone gel wells, with suture anchors. 


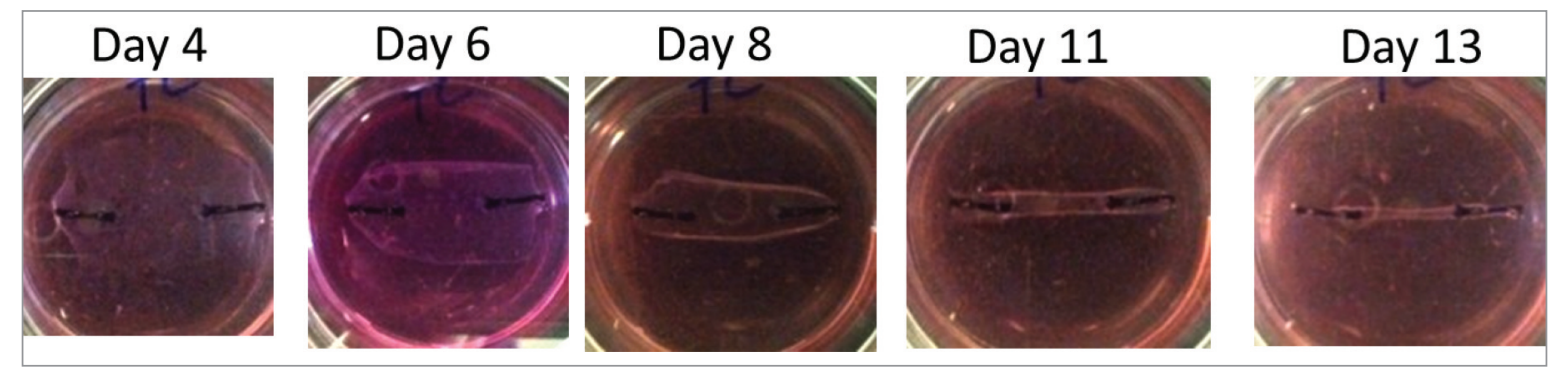

Figure 2. Top view of actual tissue engineered construct contracting around suture anchors over time on silicone gel well.

at $37^{\circ} \mathrm{C}$. After 2 weeks, the TECs were harvested for response measures.

\section{Immunohistochemistry}

TECs were fixed in $10 \%$ neutral buffered formalin for 1 hour, frozen in optimal cutting temperature (OCT) embedding media, and then sectioned in a serial fashion $(7-8 \mu \mathrm{m})$ at 3 levels along the construct height (superficial-middle-deep) to yield 3 levels which would be averaged together for each technical replicate. Construct sections were stained with anticollagen I (Col1) and anti-collagen III (Col3) antibodies and counterstained with DAPI.

\section{Image Analysis}

Tiled images (10X objective) of the TEC were acquired and a region of interest was drawn around the gel perimeter. Each channel was the sholded equally to create binary images, the positive pixels were counted, and converted to unit area $\left(\mathrm{mm}^{2}\right)$, using Fiji image analysis software 25 . The staining values were normalized to total TEC area or total cell number to account for variability in gel compaction and proliferation.

\section{Results}

Raw Type I and III collagen staining Low oxygen culture and TGF $\beta 3$ both demonstrated a positive effect on type I and III collagen staining relative to negative control groups both individually and combined. In addition, it was noted that TGF $\beta 3$ addition in low-oxygen conditions produced a greater increase in type I and III collagen staining relative to normal oxygen conditions (Fig. 3).

Type I and III Collagen Staining Normalized to Construct Area

Due to variations in size of TEC constructs, type I and III collagen staining was normalized to the area of the gel. Low oxygen culture and TGF 33 both demonstrated a positive effect on type I and III collagen staining
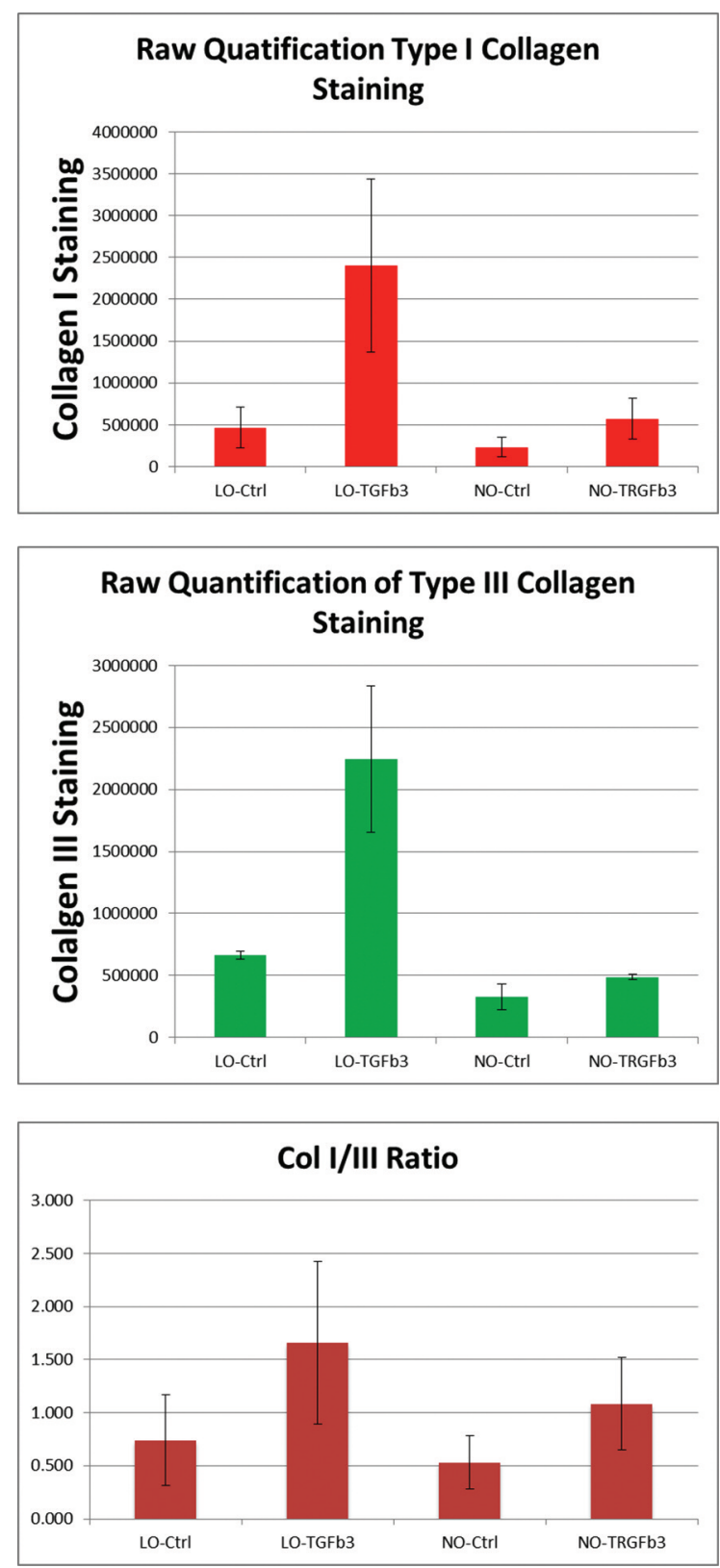

Figure 3. Graph depiction of raw pixel counts of type I and III collagen staining within TEC construct. 

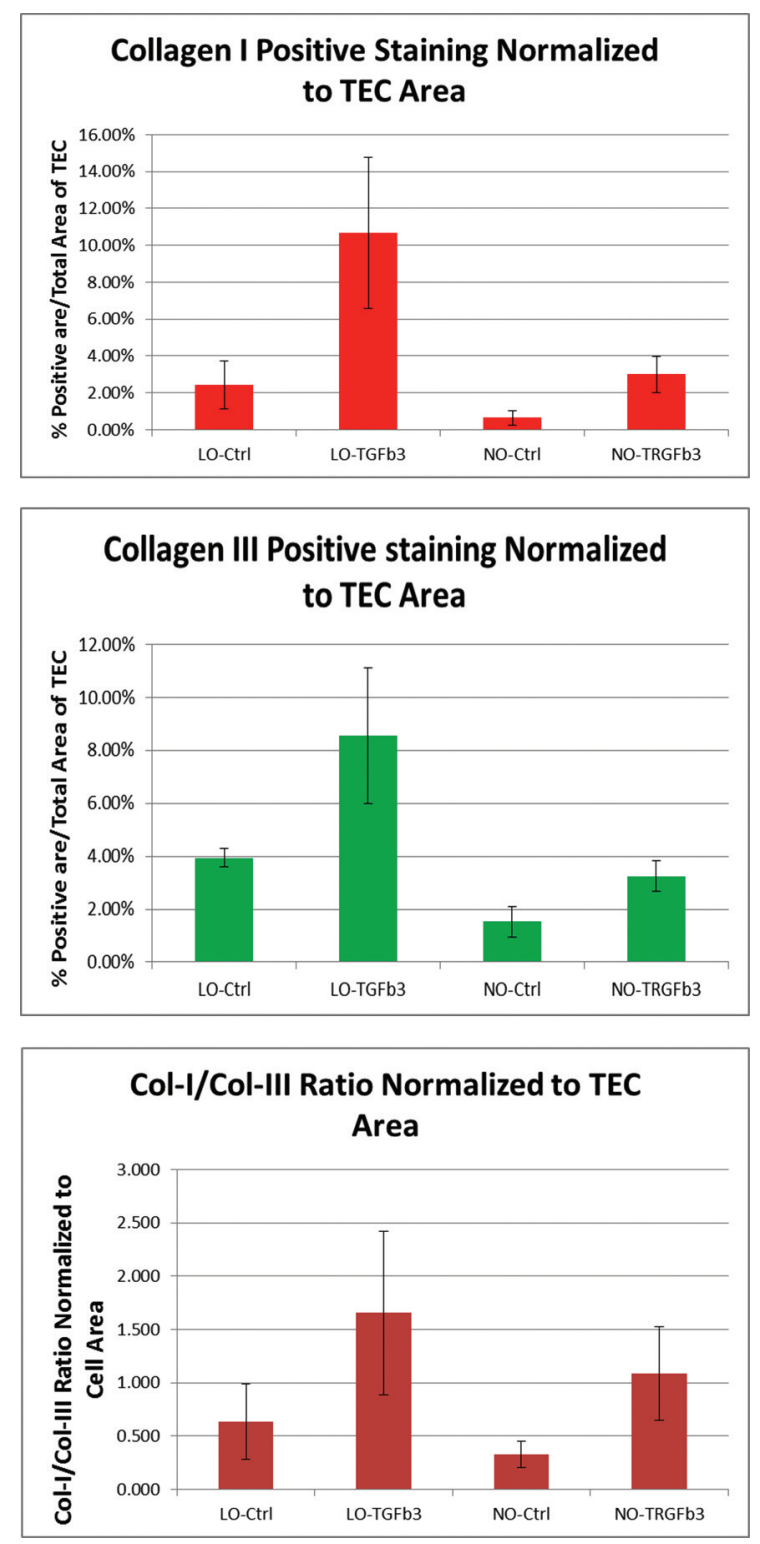

Figure 4. Graph depiction of pixel counts of type I and III collagen staining within TEC construct when normalized to TEC area.

relative to negative control groups both individually and combined (Fig. 4).

Type I and III Collagen Staining Normalized to Cell Number

Due to an appreciable increase in cell number collagen staining was again normalized to cell number to elucidate extracellular matrix production on a cell-bycell basis. Low oxygen culture and TGF $\beta 3$ both demonstrated a positive effect on type I and III collagen staining relative to negative control groups both individually and combined (Fig. 5). However, in normal oxygen conditions, TGF $\beta 3$ did not produce a net effect on type III collagen production.

\section{Discussion}

The purpose of this paper was to investigate the expression of hMSC extracellular matrix proteins, proliferation, and rate of gel construct compaction in a 3-dimensional environment in vitro, in response to elements that may affect their differentiation in the clinical setting. Variables included low oxygen tension and TGF $\beta 3$ growth factor, as previous studies have shown these two variables to have significant effects on the lineage propensities of the hMSC cell type ${ }^{13,27-29}$.

In this study, we showed that hMSCs are clearly influenced by TGF $\beta 3$ and low oxygen culture conditions, both separately and together. Furthermore, we found that type I and III collagen extracellular matrix proteins are positively influenced by these variables, indicating that they may be associated with regulation of hMSC lineage tendencies.

There is a number of studies to date which have investigated the nature of TGF $\beta 3$ influence on hMSCs. In the adult, healing tendon has been shown to have type I and III collagen, however the major constituent of mature, uninjured tendon is primarily type I collagen ${ }^{30}$. Kapacee et al. ${ }^{9}$ performed a study which demonstrated type I collagen was synthesized in TECs when in the presence of TGF $\beta 3$ growth factor. Multiple studies investigating TGF $\beta 3$ in tendon healing have shown similar results, implicating TGF $\beta 3$ with the formation of mature tendon composed primarily of type I collagen, as opposed to TGF $\beta 1$ being associated with scar-based healing 29,31 .

Our results parallel findings which suggest TGF $\beta 3$ may play a role in the regulation of collagen fibril synthesis, with emphasis on type I collagen ${ }^{13,14,28,29}$. In the presence of TGF $\beta 3$, we showed an increase in both type I and III collagen. However, our data further demonstrated an increase in type I:type III collagen ratios, indicating type I collagen expression is more positively influenced than type III collagen in the presence ofTGF 33 signaling. This trend was preserved in the setting of low oxygen culture.

The effect of TGF $\beta 3$ and low oxygen on the type I:III collagen ratio was of particular interest in this study. It has been shown in previous biomechanical studies that mature tendon composed of type I collagen has higher load-to-failure rates than surgically repaired tendon composed mainly of scar. As a result, a theoretical benefit may be elicited with the application of hMSCs to a tendon injury site when in the in vivo environment, as this could aim to promote type I collagen synthesis over scar based healing.

A number of in vitro studies has investigated the behavior of hMSCs and progenitor cells of their lineage in low oxygen culture conditions ${ }^{16,24}$. However, there are no studies to-date investigating the effect of oxygen levels on hMSCs and expression of tendon lineage markers, as the literature currently focuses on bone and cartilage, with results remaining inconclusive ${ }^{17,21,32}$. Lower oxygen tension with hMSC culture has been shown in a number of investigations to maintain their progenitor state, and appears to regulate their proliferation, viability, and their commitment to differentiation ${ }^{17,23,24}$. Malla- 

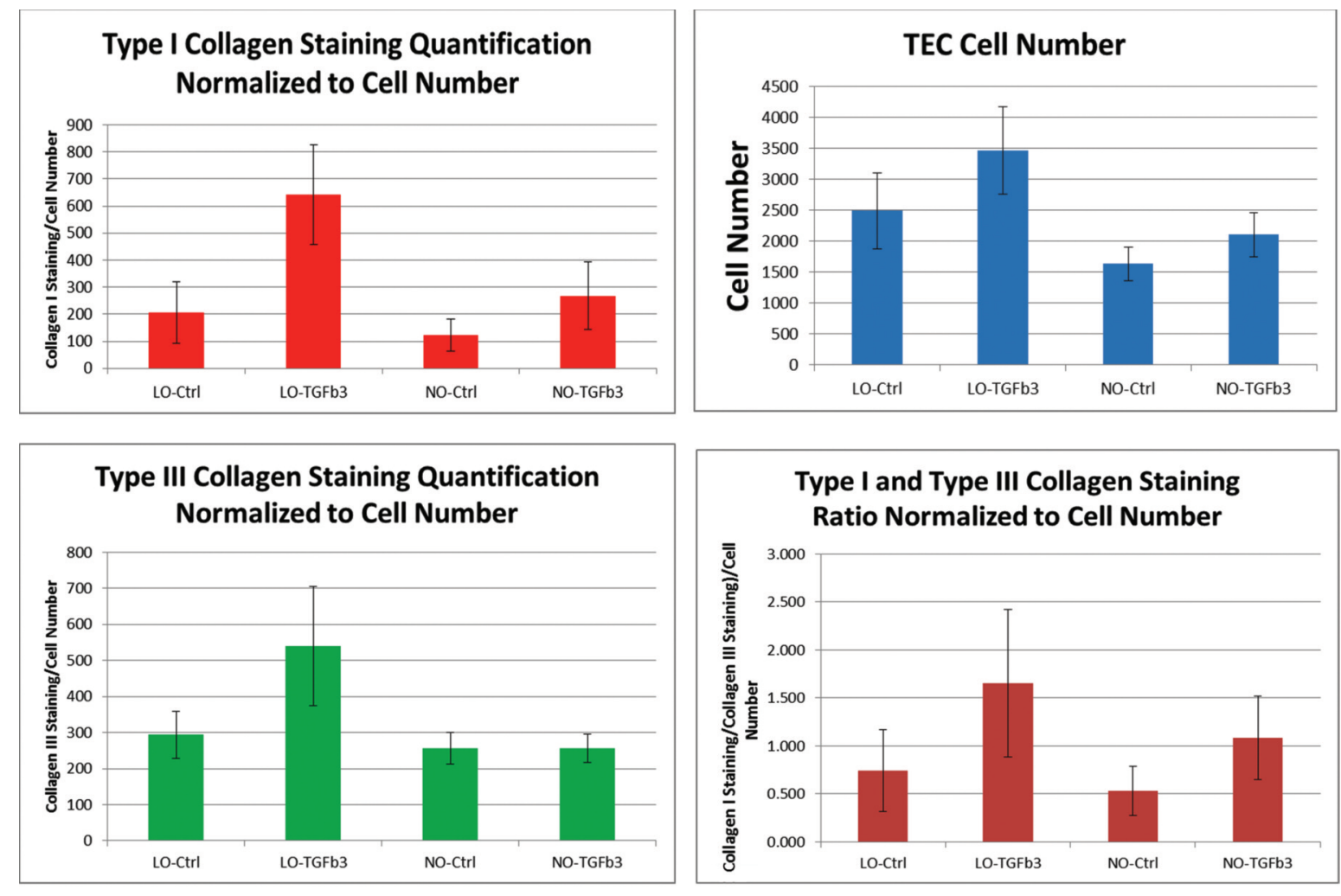

Figure 5. Graph depiction of pixel counts of type I and III collagen staining within TEC construct when normalized to TEC area.

di et al. ${ }^{17}$ showed that low oxygen tension diminished chondrogenesis and osteogenesis in relation to ambient oxygen conditions.

In our study, low oxygen demonstrated an effect on hMSCs in a way that parallels previous literature and played a clear role in the regulation of extracellular matrix proteins in the 3-dimensional culture environment. Our data also suggests that synthesis of collagenous proteins is positively influenced in the setting of lower oxygen conditions, relative to ambient oxygen conditions. In addition, type I collagen synthesis seems to be more positively influenced, relative to type III collagen, similar to the effect seen with TGF $\beta 3$ growth factor addition.

These findings are encouraging when considering the applicability of hMSCs to injured or surgically repaired tendon in the clinical setting, as our data may suggest that in vivo oxygen conditions may alter the lineage propensities of this cell type in a beneficial manner. This being in regards to both an overall increase in the rate of collagen production, but in particular, an increase in the type I:III collagen ratio. Further study is warranted in this area to elucidate the best efficacy and safety of this cell population as it may be used in this setting.

Our results may also be relevant to the field of functional tissue engineering. Currently there are three primary components identified for successful creation of new tissues; cell source, scaffolds, and bioactive factors ${ }^{33}$. A number of studies have shown cyclic mechanical strain to be a potent bioactive factors for tenogenesis, demonstrating a positive influence on MSC expression of tenogenic markers ${ }^{34,35}$. The findings of this paper similarly infer that low oxygen conditions and TGF $\beta 3$ may in fact be crucial bioactive factors for collagen expression, and while further study is needed, may improve biomechanical properties of tenogenic tissue engineered in vitro for the purposes of functional tissue engineering.

It is widely accepted that the developmental fate hMSC progenitor cells are heavily influenced by numerous environmental factors ${ }^{36}$. However, the manner in which these cells are influenced, and what factors they are influenced by remains a topic of study and debate. With current technologies aiming to use these progenitor cells in a clinical setting, it may prove beneficial for these factors and behaviors to be more thoroughly elucidated with in vitro and in vivo analysis.

\section{Conclusion}

Low oxygen and TGF $\beta 3$ demonstrated a positive effect on cell number, and type I and III collagen expression in 3D culture environments. In addition, it was shown that both variables, together and separate produced an increase in the type I:III collagen ratio. The results of this study may indicate that low oxygen culture conditions may have a significant effect on hMSC lineage propensities which may prove to be an important consideration for future in vitro studies. 
Mesenchymal stem cell response to growth factor treatment and low oxygen tension in 3-dimensional construct environment

\section{References}

1. Butler DL, Gooch C, Kinneberg KR, et al. The use of mesenchymal stem cells in collagen-based scaffolds for tissue-engineered repair of tendons. Nat Protoc. 2010;5(5):849-863.

2. van der Meijden OA, Westgard P, Chandler Z, Gaskill TR, Kokmeyer D, Millett PJ. Rehabilitation after arthroscopic rotator cuff repair: current concepts review and evidence-based guidelines. Int J Sports Phys Ther. 2012;7(2):197-218.

3. Mazzocca AD, McCarthy MB, Chowaniec DM, Cote MP, Arciero RA, Drissi H. Rapid isolation of human stem cells (connective tissue progenitor cells) from the proximal humerus during arthroscopic rotator cuff surgery. Am J Sports Med. 2010;38(7):1438-1447.

4. Beitzel K, McCarthy MB, Cote MP, et al. Rapid isolation of human stem cells (connective progenitor cells) from the distal femur during arthroscopic knee surgery. Arthroscopy. 2012;28 (1):74-84.

5. Butler DL, Juncosa-Melvin N, Boivin GP, et al. Functional tissue engineering for tendon repair: A multidisciplinary strategy using mesenchymal stem cells, bioscaffolds, and mechanical stimulation. J Orthop Res. 2008;26(1):1-9.

6. Galatz LM, Sandell LJ, Rothermich SY, et al. Characteristics of the rat supraspinatus tendon during tendon-to-bone healing after acute injury. J Orthop Res. 2006;24(3):541-550.

7. Kovacevic $D$, Rodeo SA. Biological augmentation of rotator cuff tendon repair. Clin Orthop Relat Res. 2008;466(3):622-633.

8. Manning CN, Kim HM, Sakiyama-Elbert S, Galatz LM, Havlioglu N, Thomopoulos S. Sustained delivery of transforming growth factor beta three enhances tendon-to-bone healing in a rat model. J Orthop Res. 2011;29(7):1099-1105.

9. Kapacee Z, Yeung CY, Lu Y, Crabtree D, Holmes DF, Kadler $\mathrm{KE}$. Synthesis of embryonic tendon-like tissue by human marrow stromal/mesenchymal stem cells requires a three-dimensional environment and transforming growth factor beta3. Matrix Biol. 2010;29(8):668-677.

10. Deans RJ, Moseley AB. Mesenchymal stem cells: biology and potential clinical uses. Exp Hematol. 2000;28(8):875-884 .

11. Via AG, Frizziero A, Oliva F. Biological properties of mesenchymal Stem Cells from different sources. Muscles Ligaments Tendons J. 2012;2(3):154-162.

12. Mazzocca AD, McCarthy MB, Chowaniec $D$, et al. Bone marrow-derived mesenchymal stem cells obtained during arthroscopic rotator cuff repair surgery show potential for tendon cell differentiation after treatment with insulin. Arthroscopy. 2011;27(11):1459-1471.

13. Hosokawa R, Nonaka K, Morifuji M, Shum L, Ohishi M. TGFbeta 3 decreases type I collagen and scarring after labioplasty. J Dent Res. 2003;82(7):558-564.

14. Bayer ML, Yeung CY, Kadler KE, et al. The initiation of embryonic-like collagen fibrillogenesis by adult human tendon fibroblasts when cultured under tension. Biomaterials. 2010;31(18): 4889-4897.

15. Pryce BA, Brent AE, Murchison ND, Tabin CJ, Schweitzer R. Generation of transgenic tendon reporters, ScxGFP and ScxAP, using regulatory elements of the scleraxis gene. Dev Dyn. 2007;236(6):1677-1682.

16. Buravkova LB, Anokhina EB. Mesenchymal stromal progenitor cells: general characteristics and functional state in low oxygen tension. Ross Fiziol Zh Im I M Sechenova. 2008;94(7):737-757.

17. Malladi $\mathrm{P}, \mathrm{Xu} \mathrm{Y}, \mathrm{Chiou} \mathrm{M}$, Giaccia AJ, Longaker MT. Effect of reduced oxygen tension on chondrogenesis and osteogenesis in adipose-derived mesenchymal cells. Am J Physiol Cell Physiol. 2006;290(4):C1139-1146.

18. Kofoed H, Sjontoft E, Siemssen SO, Olesen HP. Bone marrow circulation after osteotomy. Blood flow, pO2, pCO2, and pressure studied in dogs. Acta Orthop Scand. 1985;56(5):400-403.

19. Grant JL, Smith B. Bone marrow gas tensions, bone marrow blood flow, and erythropoiesis in man. Ann Intern Med. 1963:58:801-809.

20. Tondevold E, Eriksen J, Jansen E. Observations on long bone medullary pressures in relation to arterial $\mathrm{PO} 2, \mathrm{PCO} 2$ and $\mathrm{pH}$ in the anaesthetized dog. Acta Orthop Scand. 1979;50(6 Pt 1):645-651.

21. Lennon DP, Edmison JM, Caplan Al. Cultivation of rat marrowderived mesenchymal stem cells in reduced oxygen tension: effects on in vitro and in vivo osteochondrogenesis. J Cell Physiol. 2001;187(3):345-355.

22. Chow DC, Wenning LA, Miller WM, Papoutsakis ET. Modeling $\mathrm{pO}(2)$ distributions in the bone marrow hematopoietic compartment. II. Modified Kroghian models. Biophys J. 2001;81 (2):685-696.

23. Buravkova LB, Grinakovskaia OS, Andreeva EP, Zhambalova AP, Kozionova MP. Characteristics of human lipoaspirate-isolated mesenchymal stromal cells cultivated under a lower oxygen tension. Tsitologiia. 2009;51(1):5-11.

24. D'Ippolito G, Diabira S, Howard GA, Roos BA, Schiller PC. Low oxygen tension inhibits osteogenic differentiation and enhances stemness of human MIAMI cells. Bone. 2006;39(3): 513-522.

25. Padulo J, Oliva F, Frizziero A, Maffulli N. Muscle, Ligaments and Tendons Journal. Basic principles and recommendations in clinical and field science research. MLTJ. 2013;4:250-252.

26. Schindelin J, Arganda-Carreras I, Frise E, et al. Fiji: an opensource platform for biological-image analysis. Nat Methods. 2012;9(7):676-682.

27. Fehrer $\mathrm{C}$, Brunauer R, Laschober $\mathrm{G}$, et al. Reduced oxygen tension attenuates differentiation capacity of human mesenchymal stem cells and prolongs their lifespan. Aging Cell. 2007;6(6):745-757.

28. Kim HJ, Kang SW, Lim HC, et al. The role of transforming growth factor-beta and bone morphogenetic protein with fibrin glue in healing of bone-tendon junction injury. Connect Tissue Res. 2007;48(6):309-315.

29. Kim HM, Galatz LM, Das R, Havlioglu N, Rothermich SY, Thomopoulos $\mathrm{S}$. The role of transforming growth factor beta isoforms in tendon-to-bone healing. Connect Tissue Res. 2011;52(2):87-98.

30. Williams IF, McCullagh KG, Silver IA. The distribution of types I and III collagen and fibronectin in the healing equine tendon. Connect Tissue Res. 1984;12(3-4):211-227.

31. Dahlgren LA, Mohammed HO, Nixon AJ. Temporal expression of growth factors and matrix molecules in healing tendon lesions. J Orthop Res. 2005;23(1):84-92.

32. Malda J, Martens DE, Tramper J, van Blitterswijk CA, Riesle J. Cartilage tissue engineering: controversy in the effect of oxygen. Crit Rev Biotechnol. 2003;23(3):175-194.

33. Ohba S, Hojo H, Chung Ul. Bioactive factors for tissue regeneration: state of the art. Muscles Ligaments Tendons $\mathrm{J}$. 2012;2(3):193-203.

34. Delaine-Smith RM, Reilly GC. Mesenchymal stem cell responses to mechanical stimuli. Muscles Ligaments Tendons J. 2012;2(3):169-180.

35. Park JS, Chu JS, Cheng C, Chen F, Chen D, Li S. Differential effects of equiaxial and uniaxial strain on mesenchymal stem cells. Biotechnol Bioeng. 2004;88(3):359-368.

36. Ankeny DP, McTigue DM, Jakeman LB. Bone marrow transplants provide tissue protection and directional guidance for axons after contusive spinal cord injury in rats. Exp Neurol. 2004;190(1):17-31 